\title{
Editorial: Flow and transport in porous media in the face of uncertainty, part I
}

Kok-Kwang Phoon

Distinguished Professor,Department of Civil and Environmental Engineering, National University of Singapore, Singapore (kkphoon@nus.edu.sg)

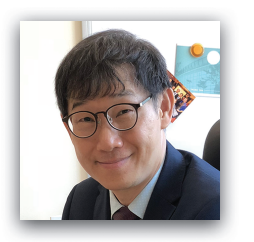

The ICE State of the Nation 2017 Report defined digital transformation as the application of digital technologies to all aspects of human life ... It also refers to the transformation of how we value data, and the impacts upon processes and systems, and ultimately decision making' (ICE, 2017). While our geotechnical engineering profession has been very successful in making decisions in the presence of extremely limited data, clever modelling and engineering judgment, this strategy is now fundamentally out of alignment with broad sweeping trends disrupting all industries. In particular, this strategy does not encourage monetizing and collection of data in innovative ways that can transform processes and systems, and ultimately decision making in every aspect of geotechnical engineering from design, build, operate, maintain to decommission. The four main characteristics of data in the data science literature are: volume, variety, velocity and veracity. Veracity or 'data in doubt' imposes a fundamental limit on the value of data to decision making. In geotechnical engineering it includes two types of uncertainties, namely the aleatoric uncertainty (uncertainty arising from spatial variability) and epistemic uncertainty (uncertainty arising from insufficient or a lack of knowledge). This themed issue deals with flow and other related transport problems in porous media, with emphasis on how uncertainties in hydraulic properties are characterized and how these uncertainties impact engineering decisions. The Guest Editor believes such approaches will better manifest the value of data and bring geotechnical engineering into better alignment with the new data-centric economy.

There are numerous challenging issues in this important class of problems. Hydraulic properties are intrinsically uncertain and spatially variable because they are related to the pore size distribution and other aspects of soil structure. The impact of input uncertainty on the flow/transport regime is significant for at least four reasons. First, the uncertainties associated with hydraulic properties are known to be the highest and most difficult to characterize, among uncertainties associated with other soil properties. Second, the flow/ transport regime may not depend on spatially averaged properties. For example, a thin connected seam of highly permeable soil can be pivotal in a flow problem. Phenomena that depend on spatially averaged properties enjoy a reduction in uncertainty in the response. Third, flow/transport in unsaturated or engineered soils can be a highly nonlinear function of hydraulic and other properties which magnify the input uncertainty and results in responses following strongly non-normal probability distributions. Finally, the presence of spatial variability can produce qualitatively new mechanisms not observable in a conventional homogeneous medium. This impact is arguably more fundamental than an assessment of the probability of failure and not well appreciated in the deterministic community where only homogeneous or simple layered systems are considered. Some basic aspects have been covered (ASCE, 2016a, 2016b) but there are other dimensions explored by the ten papers in this themed issue.

The first two papers presented statistical methods to characterize hydraulic properties. Prakash et al. (2019) proposed a novel method to estimate soil suction in natural fibre reinforced soil by using the crack intensity factor (CIF). The CIF can be obtained from images and thus opening the way for fast mapping of soil suction over large areas using drones. The statistical relationships between CIF and soil suction for bare soil and three soil-fibre composites using natural fibres (coir, jute and water hyacinth) were established using copulas. Zhai et al. (2019) proposed a new model to quantify the hysteresis of soil-water characteristics curve (SWCC) due to the ink bottle effect and the entrapped air. The uncertainty in the estimated wetting SWCC was characterized based on the uncertainty in the pore size distribution function and the variability in the entrapped air. The model (including its uncertainty) was shown to be reasonable for sandy and silty soils. Its application to clayey soil needs further study.

The following three papers studied various contaminant transport problems with the impact of uncertainty considered in two papers. Elder and Benson (2019) compared the performance and cost for horizontal permeable reactive barriers (HPRBs), funnel and gate permeable reactive barriers (FGPRBs) and caisson permeable reactive barriers (CPRBs) in spatially variable aquifers that achieve the same goal. The aquifers are simulated using a lognormal random field for the hydraulic conductivity with geometric mean 
of $2.8 \times 10^{-5} \mathrm{~m} / \mathrm{s}$ and $\log$-standard deviation (roughly equal to coefficient of variation) of $0 \cdot 5,1 \cdot 5$, and $3 \cdot 0$. These three values produces a near uniform, moderately variable and highly variable aquifer, respectively. It was found that FGPRB is less expensive than the CPRB for all levels of aquifer spatial variability. The HPRB is the most economical choice only in near uniform aquifers. Hong and Shackelford (2019) assessed the effect of a mixed salt solution (potassium chloride and zinc chloride) on the migration behaviour of both metals through zeolite-amended soil-bentonite backfills. From long-term column tests, it was found that the transport of chloride, potassium and zinc was diffusion dominated. In addition, zeolite-amended backfills enhanced retention of metals relative to that for the unamended backfill and the effect of metals competition appeared minimal based on the conditions imposed for the column tests. Nazeeh and Sivakumar Babu (2019) presented an interesting case study of the transport of radionuclide caesium through a near surface disposal facility to an underlying aquifer. The random variables considered are (1) bulk density, porosity, ground water velocity, dispersivity and distribution coefficient of the aquifer, and (2) diffusion coefficient of the waste container. Subset simulation reveals that the probability of failure (dose rate higher than permissible limit) is mainly dependent on the diffusion coefficient below the disposal area and the ground water velocity beyond the disposal area.

Making risk-informed decision in the face of uncertainty is the recommended approach (ISO, 2015) and the papers in this themed issue contribute to this decision making process. Nonetheless, even this general approach should be situated within the evolving needs of Industry 4.0. Insofar as monetizing our data estate, one can envisage at least four future research needs. (1) extraction, refinement, distribution, and value creation of geotechnical information from its original form: Multivariate, Uncertain and Unique, Sparse, Incomplete, and potentially Corrupted (MUSIC) (or MUSIC-X denoting MUSIC data that vary along spatial and/or temporal dimension (X) - it is the 'new oil' (2) exploitation of novel sensing methods such as wireless sensor network or drone swarm, (3) development of physics-informed and/or purely data-driven learning approaches to support explainable decision making and (4) the role of engineering judgment in smart asset management that includes automation.

The Guest Editor would like to extend his gratitude to the invited authors for their technical contributions that further our understanding of this challenging class of problems.

\section{REFERENCES}

ASCE (American Society of Civil Engineers) (2016a) ASCE/EWRI 50-08: Standard Guideline for Fitting Saturated Hydraulic Conductivity Using Probability Density Function and Standard Guideline for Calculating the Effective Saturated Hydraulic Conductivity. American Society of Civil Engineers, Reston, VA, USA.

ASCE (American Society of Civil Engineers) (2016b) ASCE/EWRI 54-10: Standard Guideline for the Geostatistical Estimation and Block-Average of Homogeneous and Isotropic Saturated Hydraulic Conductivity. American Society of Civil Engineers, Reston, VA, USA.

Elder CR and Benson CH (2019) Performance and economic comparison of PRB types in heterogeneous aquifers. Environmental Geotechnics 6(4): 214-224, https://doi.org/10.1680/jenge.17.00063.

Hong CS and Shackelford CD (2019) Mixed metals migration through zeolite-amended backfills for vertical cut-off walls. Environmental Geotechnics 6(4): 225-241, https://doi.org/10.1680/jenge.17.00041.

ICE (Institute of Civil Engineers) (2017) ICE State of The Nation 2017: Digital Transformation. ICE, London, UK. See https://www.ice.org. uk/getattachment/news-and-insight/policy/state-of-the-nation-2017digital-transformation/ICE-SoN-Report-Web-Updated.pdf.aspx (accessed 16/03/2019).

ISO (International Organization for Standardization) (2015) ISO 2394:2015: General principles on reliability for structures. International Organization for Standardization, Geneva, Switzerland. Nazeeh KM and Sivakumar Babu GL (2019) Reliability analysis of nearsurface disposal facility using subset simulation. Environmental Geotechnics 6(4): 242-249, https://doi.org/10.1680/jenge.17.00004.

Prakash A, Bordoloi S, Hazra B et al. (2019) Probabilistic analysis of soil suction and cracking in fibre-reinforced soil under drying-wetting cycles in India. Environmental Geotechnics 6(4): 188-203, https://doi. org/10.1680/jenge.18.00067.

Zhai P, Rahardjo H and Satyanaga A (2019) Uncertainty in the estimation of hysteresis of soil-water characteristic curve. Environmental Geotechnics 6(4): 204-213, https://doi.org/10.1680/jenge.17.00008. 